\title{
Syringoperitoneal Shunt for Syringomyelia: A Preliminary Report
}

Tim W. Phillips, M.D., and Glenn W. Kindt, M.D.

Syringoperitoneal shunting has been used in the treatment of 4 patients with idiopathic syringomyelia. The procedures have been performed without morbidity or mortality. Postoperative observation, ranging from 7 to 23 months, has not revealed progression of symptoms or failure of the shunt. The pathophysiological theories of syringomyelia are discussed, and various surgical procedures for syringomyelia are reviewed and their results compared to the effectiveness of the syringoperitoneal shunt.

Phillips TW, Kindt GW: Syringoperitoneal shunt for syringomyelia: a preliminary report. Surg Neurol $16: 462-466,1981$

The term syringomyelia was first coined in 1837 by Oliver from the Greek roots meaning "to become hollow" and "marrow" [6]. Ever since the first case reported by Etienne in 1564 [6], the etiology, the pathophysiology, and the most prudent form of treatment have remained controversial. As is common with numerous idiopathic disorders in medicine, the treatment approaches that have been proposed and advocated are widely varied. Surgical treatment has included direct syrinx decompression, shunting procedures, and posterior fossa reconstruction. A few of these procedures are fraught with high rates of morbidity and mortality, while others, though representing little risk, have not always given encouraging results [25]. Consequently, no individual technique has enjoyed a large number of proponents.

Our current experience in the treatment of syringomyelia results from the use of a syringoperitoneal shunt. The primary goal in performing this procedure is to provide a means to ensure continuous drainage of the contents of the syrinx. Additionally, syringoperitoneal shunting offers little potential risk to patients and requires no unusual equipment or materials.

\section{Method}

Technically, the operating procedure is quite straightforward. The patient is placed in a prone position and a two-

From the Department of Surgery, Section of Neurosurgery, University of Michigan, Ann Arbor, MI.

Address reprint requests to Dr. Tim W. Phillips, Department of Surgery (Neurosurgery), University of Michigan Hospital, Ann Arbor, MI 48109.

Key words: syringomyelia; syringoperitoneal shunt; shunt. level or three-level laminectomy is performed over the syrinx cavity, which has been previously defined by myelography or computed tomography. The dura mater is opened and under magnification a 3 to $4 \mathrm{~mm}$ myelotomy is performed just medial to the dorsal roots, where, in our experience, the syrinx wall is thinnest. A ventricular catheter made of silicone rubber, with an inside diameter of $1.5 \mathrm{~mm}$, an outside diameter of $3.1 \mathrm{~mm}$, and multiple side holes, is advanced into the syrinx. The catheter is then secured to the dura mater and the dural incision is closed. An incision is then made on the right side of the patient's abdomen, although an incision can be made on the left if a previous operation or the patient's condition makes a right-sided incision undesirable. An appropriate length of connecting catheter is passed subcutaneously from the right abdominal incision to the midline incision for the laminectomy, where the two catheters are joined. An extremely low-pressure valve is attached to the connecting catheter within the right abdominal incision, and the laminectomy incision is closed. The patient is then turned to a lateral decubitus position so that a third skin incision can be made in a paramedian fashion to enter the peritoneal cavity. Through that paramedian abdominal incision, a small incision is made in the peritoneum so that 20 to $30 \mathrm{~cm}$ of the silicone rubber peritoneal catheter, with an inside diameter of $1.2 \mathrm{~mm}$ and an outside diameter of 2.5 $\mathrm{mm}$, can be advanced into the peritoneal cavity. An absorbable pursestring suture is placed in the peritoneum around the peritoneal catheter to secure its position. The distal portion of the peritoneal catheter is subsequently passed subcutaneously from the paramedian abdominal incision to the right-sided abdominal incision, where the peritoneal catheter is attached to the valve. The operation is completed by closing the skin incisions (see Fig. 1).

\section{Case Reports}

Between December, 1978, and February, 1980, 4 patients underwent syringoperitoneal shunting procedures for their syringomyelia, which had been diagnosed by clinical examination, myelography, and computed tomography. The patients had been symptomatic for two to eleven years. A lumbar laminectomy for aspiration of the cyst had been performed in 1 patient seven months prior to syringoperitoneal shunting. All patients have been subsequently observed by the neurosurgery service for periods of time ranging from seven to twenty-two months. 


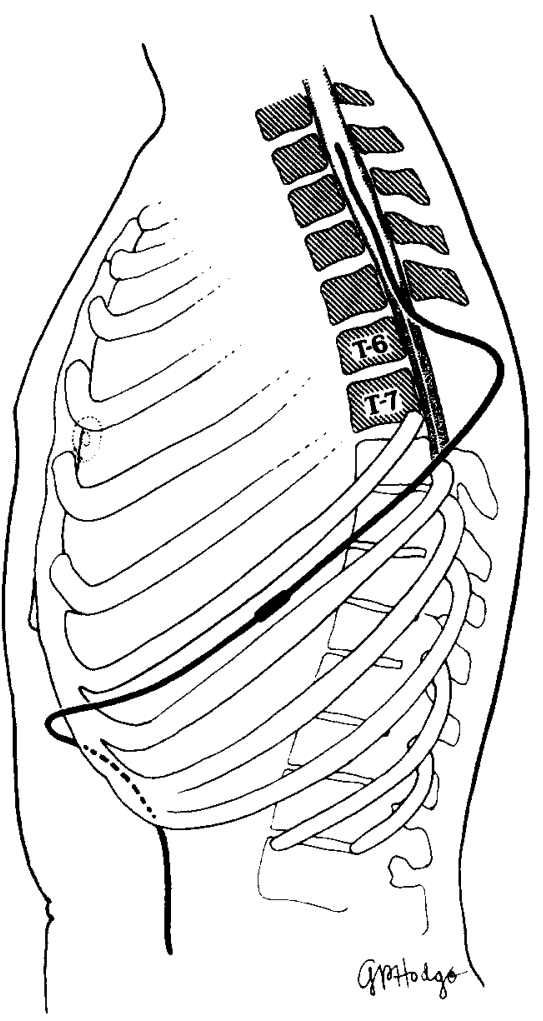

Fig. 1. A syringoperitoneal shunt in place.

Two patients had a complete reversal of their preoperative motor deficits, another patient demonstrated improvement in motor strength, and the fourth patient demonstrated no significant change from the preoperative state. Prior to shunting, all patients reported that their symptoms were periodically progressive. After shunting, no complaints of progressive symptoms were noted. By objective examination, neurological deficits have not progressed. There has been no morbidity or mortality from the placement or the presence of the syringoperitoneal shunt.

\section{Patient 1}

A 56-year-old woman first became symptomatic in 1972 . Her initial symptom was pain radiating to her right shoulder, arm, and hand. The symptoms progressed to include pain in the right leg and weakness in the distal right leg. She was initially evaluated in her native country in Latin America and subsequently referred to the University of Michigan. At the time of admission to the University of Michigan Hospital on December 6, 1979, her mental status and cranial nerves were normal. Her right tibialis anterior strength was $3 / 5$ and the remainder of the motor examination was normal. Sensory testing demonstrated diminished pinprick and temperature sensation distal to the T4 dermatome on the right with sacral sparing and in the T3 through T5 dermatomes on the left. Vibratory sensation was absent in both legs, and position sensation was intact throughout. Babinski signs were absent, and the deep tendon reflexes were unremarkable. Plain roentgenograms of her spine demonstrated scoliosis, and a complete myelog. raphy demonstrated a widened cervical and thoracic spinal cord.

On December 14, 1979, a syringoperitoneal shunt was placed without complications. During the operation it was found that clear fluid filled the syrinx, but the fluid was not sent for laboratory analysis. Following the operation her pain and sensory disturbances persisted. Her right footdrop improved and she returned to Latin America on December 29, 1979.

She has been seen once in our neurosurgery clinic eight months following the operation, at which time the preoperative sensory deficits remained unchanged. Her motor examination revealed that her right tibialis anterior strength was 5/5. She denied any new symptoms and returned to her home in Latin America. We have since communicated with her and her local physician numerous times; on evaluation twenty-two months following the operation her physician reported that she continued to have normal motor function.

\section{Patient 2}

A 28-year-old woman first noted paresthesias in her hand in 1968. She progressively developed weakness and muscular atrophy in both hands. On May 20,1979, she was admitted to the University of Michigan Hospital. Her admission neurological examination demonstrated normal cranial nerve function. The motor examination revealed $3 / 5$ strength of her right extensor pollicis longus and brevis, abductor pollicis, flexor digitorum profundus, extensor digitorum, and intrinsic musculature. Proximal testing of the right arm revealed $4 / 5$ strength of the deltoids, triceps, flexor carpi radialis, flexor carpi ulnaris, extensor carpi radialis, and extensor carpi ulnaris. The remainder of her motor examination was normal. Sensory examination demonstrated diminished sensation to pinprick, temperature, vibration, and light touch in the $\mathrm{C} 2$ through $\mathrm{C} 8$ dermatomes on the right and the $\mathrm{C} 2$ and $\mathrm{C} 3$ dermatomes on the left. Deep tendon reflexes were absent in the arms but were normal in the legs, and Babinski signs were absent. Myelography was performed and revealed a widened spinal cord from C2 to T11, but greatest from C4 to C6 (Fig. 2). The myelogram raised the question of an Arnold-Chiari type 1 malformation, but that diagnosis was not confirmed by computed tomography.

On June 5, 1979, a syringoperitoneal shunt was placed without complication. Clear fluid filled the syrinx but was not analyzed by the laboratory. Her neurological status was unchanged following the operation, and she was discharged on June 13, 1979. Postoperative observation at fourteen months revealed no change in her neurological status and no progression of her weakness. 


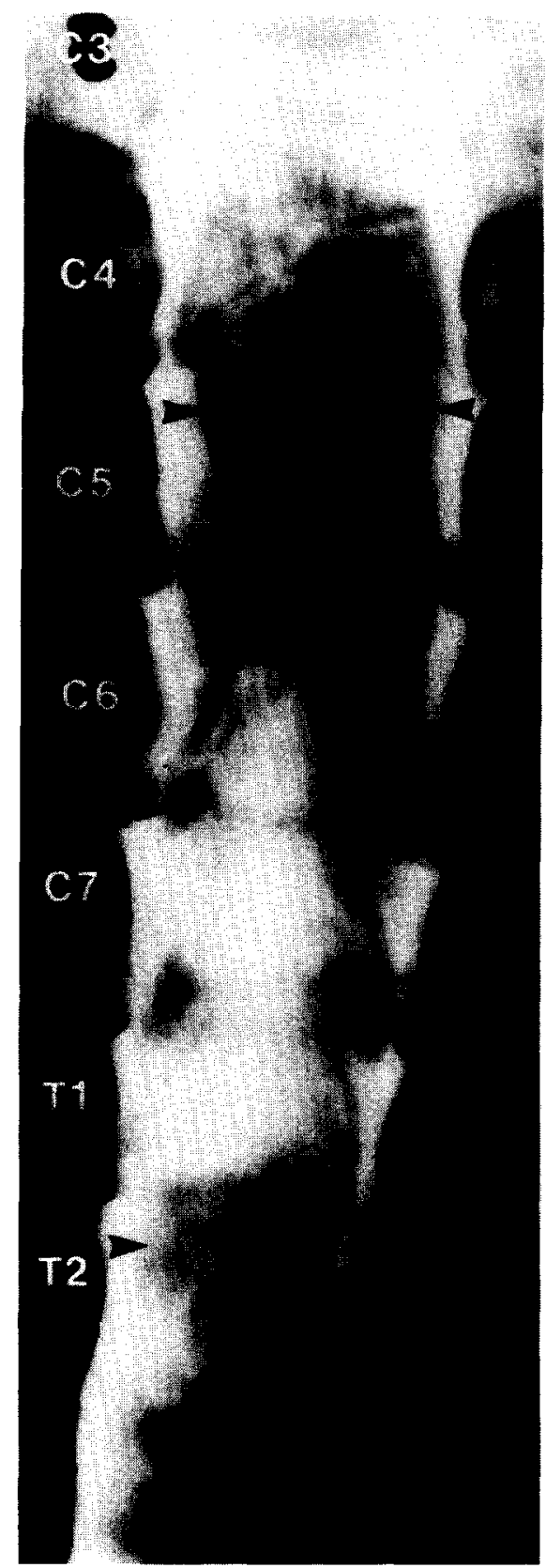

Fig. 2. Patient 2. Cervical myelogram demonstrating preoperative syringomyelia. Arrowheads outline the perimeter of a syrinx filling the cervical spinal cord.

\section{Patient 3}

A 25-year-old man first noted weakness in his right leg and arm in 1977. His weakness progressed to involve all extremities, and in May, 1979, at another hospital, he underwent a laminectomy with needle aspiration of the syrinx. His symptoms recurred and his weakness progressed following the aspiration of the syrinx. He was admitted to the University of Michigan Hospital on November 21, 1979. On admission, examination of his cranial nerves was normal. The motor examination revealed $4 / 5$ strength of the left arm. The right arm had $0 / 5$ intrinsic function and
1/5 extensor carpi ulnaris and radialis function, $1 / 5$ flexor carpi ulnaris and radialis function, $3 / 5$ tricep function, $4 / 5$ bicep function, and $4 / 5$ deltoid function. The left leg was $3 / 5$ throughout, and his right leg was noted to be completely paralyzed. Examination revealed markedly diminished pinprick, temperature, and position sensations in dermatomes $\mathrm{C} 5$ through $\mathrm{C} 7$ bilaterally and distal to $\mathrm{T} 6$ on the left. Deep tendon reflexes were hyperactive in the leg and normal in the arm. A Babinski sign was present on the left. Complete myelography demonstrated a dilated spinal cord from the foramen magnum to the conus medullaris.

On December 6, 1979, a syringoperitoneal shunt was placed without complication. Xanthochromic fluid filled the syrinx but was not sent for examination by the laboratory. A small biopsy of the syrinx wall was taken and found to be negative for neoplasm or inflammation. Following the operation the patient noted subjective improvement of the strength of both his hands. Twelve months after the operation, the minimal improvement in strength of his hands continued. His sensory examination remained unchanged, and he denied progression of other symptoms.

\section{Patient 4}

A 32-year-old woman first noted weakness in her right arm in 1977. These symptoms progressed to include numbness in her right face, neck, and upper thorax. She was admitted to the University of Michigan Hospital on January 8, 1980, for evaluation of her symptoms. Examination of the cranial nerves demonstrated decreased pinprick sensation in the right trigeminal distribution. Motor examination demonstrated $4 / 5$ strength in her right deltoid, bicep, tricep, extensor carpi ulnaris, extensor carpi radialis, flexor carpi ulnaris, flexor carpi radialis, intrinsic muscles of the hand, and iliopsoas. The sensory examination revealed diminished touch, pinprick, vibration, and position sensations in dermatomes $\mathrm{C} 2$ to $\mathrm{T} 4$ on the right and diminished touch and pinprick in dermatomes $\mathrm{T} 8$ to $\mathrm{S} 2$ on the left. Deep tendon reflexes demonstrated hyperreflexia of the right bicep, tricep, and brachial radialis but were otherwise normal. Babinski signs were absent. Complete myelography was performed and demonstrated a widened cord from $\mathrm{Cl}$ through $\mathrm{T} 9$, which was greatest at $\mathrm{T} 1$ through $\mathrm{T} 5$.

She was subsequently discharged and then readmitted to the hospital for a syringoperitoneal shunt. The shunt was placed without complications on February 19, 1980. Following the operation she was noted to have markedly improved motor strength and improved sensation with only a persistent sensory deficit involving the right side of her face. Evaluation seven months following the operation revealed that the small area of diminished pinprick sensation over her right face was her only neurological deficit.

\section{Discussion}

Syringomyelia is a slowly progressive chronic process that involves a central area of cavitation and gliosis within the 
spinal cord or medulla oblongata, or occasionally both. Symptoms most commonly appear between the ages of 25 and 40 [6], and the incidence is reported to be slightly greater in males than females [1]. Symptoms of the disease include muscular atrophy and sensory changes. The sensory changes most commonly involve temperature and nociceptive modalities, sparing the sensations of position, vibration, and light touch. All symptoms are progressive in nature over a variable time course [6].

Various theories have been advanced for defining the etiology and pathophysiology of syringomyelia [2-4]. Investigators have postulated that syringomyelia is secondary to a congenital disorder $[7,12]$, the result of a tumor [26], a posttraumatic event [27], or secondary to infectious disease [18]. Various theories have also been advanced to explain the pathophysiology of syringomyelia. Gardner [8, $9,11]$ has long believed that a disorder in the outflow tract of the fourth ventricle is central to the development of syringomyelia, and has advanced his "hydrodynamic" theory to explain the progressive nature of syringomyelia. The theory proposes that arterial pulsations transmitted from the choroid plexus through the ventricular system have no exit from the fourth ventricle and are subsequently transmitted down the central canal. Pulsations are said to cause progressive destruction of the vulnerable gray matter that lies in the core of the spinal cord [9].

Bernard Williams [22-24] argues that aberrancy in the posterior fossa can lead to further degeneration of the spinal cord and medulla oblongata, but he contests the hydrodynamic theory. Williams contends that congestion in the area of the foramen magnum leads to aberration in the venous system. These abnormalities enter into play during periods of increased venous pressure (e.g., during a Valsalva maneuver) with the elevated venous pressure being transmitted through the epidural-vertebral venous system and the spinal subarachnoid space to the congested region at the foramen magnum, resulting in the transmission of the pressure to the ventricular system and subsequently to the spinal cord, thus causing degeneration and cyst formation as well as increased fluid pressure within the cyst.

The various proposed etiologies have led to numerous operations for the treatment of syringomyelia $[5,15-17,19]$. Ventriculoatrial shunting is recommended to decompress the ventricular system. Exploration of the posterior fossa has been performed to (1) remove subarachnoid adhesions in order to promote cerebral spinal fluid flow from the fourth ventricle [9]; (2) occlude the central canal at its origin from the fourth ventricle in an attempt to prevent flow into the central canal and syrinx [9]; (3) decompress vascular structures in the area of the foramen magnum [25]; and (4) decompress a small posterior fossa [14]. Diverse methods of draining the syrinx that have been promoted include percutaneous syrinx aspiration [13], syrinx-spinal subarachnoid shunting [20], and terminal ventriculostomy at the level of the conus medullaris $[10,21]$. Each of the proposed procedures can be classified into one of two groups: (1) those that divert spinal fluid flow to prevent pressure pulses from entering the syrinx; and (2) those that provide a mechanism to drain the syrinx.

The standard theories of syringomyelia have been universally concerned with the fluid-filled cyst within the spinal cord or medulla oblongata. Additionally, the pressure differential between the syrinx fluid and surrounding neural tissue is an important concern. It appears intuitively obvious that any mass within the spinal cord or medulla oblongata that is under transient or continuous increased pressure would cause neural tissue dysfunction. The goals in developing the syringoperitoneal shunt were: (1) to provide for continuous drainage of large quantities of fluid from the syrinx cavity; (2) to relieve any increased pressure in the fluid within the syrinx; and (3) to accomplish the treatment of syringomyelia with minimal morbidity. Empirically, the syringoperitoneal shunt also appears to treat the pathophysiological mechanism of syringomyelia proposed by Gardner and by Williams. A syringoperitoneal shunt is capable of draining excess spinal fluid that enters the syrinx regardless of its origin. Of further import, the peritoneal cavity represents a large-volume, low-pressure reservoir that is anatomically and physiologically separate from the central nervous system and the subarachnoid space and, as such, provides an ideal drain for syrinx fluid since large volumes of fluid can be shunted from the syrinx. Thus, the potential hammering arterial pulse, or transmitted venous pulse, can be diverted from the cyst in the spinal cord. It is postulated that collapse of the cyst and diversion of the pressure pulses will prevent further degeneration of the neural tissue that surrounds the syrinx.

In the technical performance of the syringoperitoneal shunt, it is difficult to visualize significant morbidity or mortality. This advantage has not been enjoyed by a few of the previously mentioned procedures, particularly those requiring surgery of the posterior fossa $[9,25]$. Gardner [10], a former advocate of restoration of the fourth ventricle outflow tracts and occlusion of the central canal, has since recommended terminal ventriculostomy, thus avoiding the difficulties of an operation in the posterior fossa. Although any shunt system has its own inherent difficulties, making revision of individual components essential, there exists no component of the syringoperitoneal shunt for which revision would appear particularly difficult. Further, with increased availability of precise computed tomography, diagnosis of a failed syringoperitoneal shunt can be made with either individual or serial scans.

Our use of Holter* peritoneal and ventricular catheters as well as valves does not preclude the use of other ventricular peritoneal shunting systems. The need for a valve within the system can easily be challenged. Thus far it has 
been used in all patients as a barrier to any possibility of reflux of potential peritoneal fluid. Additionally, with the valve located in the flank a distance from the catheter in the syrinx, negligible pressure would be required to open the valve in the erect or prone position.

In summary, the benefits of syringoperitoneal shunting in the treatment of syringomyelia are numerous: (1) the shunt offers immediate drainage of any volume of fluid from the syrinx; (2) it is an expedient surgical procedure; (3) there is essentially no morbidity or mortality associated with it; and (4) preliminary clinical results are encouraging. To date no patient with a syringoperitoneal shunt has demonstrated progression of symptoms and 3 of 4 have demonstrated some motor improvement; moreover, there have been no complications. Undoubtedly longer follow-up and a greater number of patients will need to be operated upon before final clinical conclusions can be made. In view of the current theories of the pathophysiology of syringomyelia and the previously proposed surgical procedures, we plan to continue syringoperitoneal shunting in the treatment of syringomyelia.

The authors gratefully acknowledge the assistance of Debbie Konopacki and Peggy Hoag in the preparation of this manuscript.

\section{References}

1. Adams RD, Victor M: Principles of Neurology. New York: McGraw-Hill, 1977, pp 486-488

2. Becker DP, Wilson JA, Watson GW: The response of the central canal of the spinal cord to hydrocephalus. J Neurosurg 36:416-423, 1972

3. Bering EJ: Circulation of the cerebral spinal fluid. J Neurosurg 19:405-413, 1962

4. Conway LW: Hydrodynamic studies in syringomyelia. J Neurosurg 27:501-514, 1967

5. Faulkner K, Loew K: The surgical treatment of syringomyelia: longterm results. Acta Neurochir (Wien) 44:3-4, 215-221, 1978

6. Finlayson AI: Syringomyelia and related conditions, in Baker $A B$, Baker LH (eds): Clinical Neurology. Hagerstown, MD: Harper \& Row, 1977, pp 1-32
7. Foster JB, Hudgson P, Pearce GW: The association of syringomyelia and congenital cervicomedullary anomalies: pathologic evidence. Brain 92:325-334, 1969

8. Gardner WJ: Hydrodynamic mechanism of syringomyelia: its relationship to myelocele. J Neurol Neurosurg Psychiatry 28:247-259, 1965

9. Gardner WJ: The Dysthaphic States: from Syringomyelia to Anencephaly. Amsterdam: Excerpta Medica, 1973

10. Gardner WJ, Bell HS, Poolos PN, Dohn DF, Steinberg M: Terminal ventriculostomy for syringomyelia. J Neurosurg 46:609-617, 1977

11. Gardner WJ, McMurry FG: Non-communicating syringomyelia: a nonexistent entity. Surg Neurol 6:251-256, 1976

12. Gustafson WA, Oldberg E: Neurologic significance of platybasia. Arch Neurol Psychiatry 44:1184-1198, 1940

13. Hall P, Gadersky J, Muller J, Campbell R, Kalsbeck J: A study of experimental syringomyelia by scanning electron microscopy. Neurosurgery 1:41-47, 1977

14. Hall PV, Muller J, Campbell RL: Hydrosyringomyelia, ischemic myelopathy and syringomyelia. J Neurosurg 43:464-470, 1975

15. Hankinson J: Syringomyelia and the surgeon, in Williams D (ed): Modern Trends in Neurology. London: Butterworths, Vol 5, 1970, pp 127-148

16. Krayenbühl $\mathrm{H}$, Benine $A$ : A new surgical approach in the treatment of hydromyelia and syringomyelia. J R Coll Surg 16:147-161, 1971

17. Love JG, Olafson RA: Syringomyelia: a look at surgical therapy. J Neurosurg 24:714-718, 1966

18. McLaurin RL, Bailey OT, Schurr PH, Ingraham FD: Myelomalacia and multiple cavities of the spinal cord secondary to adhesive arachnoiditis, an experimental study. Arch Pathol 57:138-146, 1954

19. Pitts FW, Graft RA: Syringomyelia: current status of surgical therapy. Surgery 56:806-809, 1964

20. Schneider RC: Syringomyelia: personal observations concerning the neurological diagnosis and the monitoring of treatment by computerized axial tomography. Clin Neurosurg 25:96-147, 1978

21. Singounas EG, Karvounis PC: Terminal ventriculostomy in syringomyelia. Acta Neurochir (Wien) 46:293-295, 1979

22. Williams $B$ : The distending force in the production of "communicating syringomyelia." Lancet 2:189-193, 696-697, 1969

23. Williams B: Current concepts of syringomyelia. Br J Hosp Med $4: 331-342,1970$

24. Williams B: Cerebrospinal fluid pressure response to coughing. Brain 99:331-346, 1976

25. Williams B: A critical appraisal of posterior fossa surgery for communicating syringomyelia. Brain 101:223-250, 1978

26. Williams B, Timperley WR: Three cases of communicating syringomyelia secondary to midbrain glioma. I Neurol Neurosurg Psychiatry 40:80-88, 1976

27. Williams $B$, Turner $E$ : Communicating syringomyelia presenting immediately after trauma. Acta Neurochir (Wien) 24:97-106, 1971 